\title{
Morbidity profile and prescribing patterns among outpatients in a teaching hospital in Western Nepal
}

\author{
DC Lamichhane, BR Giri, OK Pathak, OB Panta, PR Shankar*
}

\begin{abstract}
Background: Recent studies on prescribing among outpatients in hospitals in Western Nepal are lacking. The main objectives of the study were to obtain information on the morbidity pattern among outpatients and to analyze prescribing using drug use indicators. Methods: A retrospective hospital record based study from 01.01.2004 to 31.12.2004 was carried out among individuals attending the outpatient department (OPD) of the Manipal Teaching hospital, Pokhara, Western Nepal. A total of 32,017 new patients attended the OPD during the study period. Systematic random sampling ( 1 in every 20 patients) was done and 1600 patients selected. After excluding patients visiting the emergency department, those who got admitted and whose records were not available, 1261 cases were analyzed. The demographic details, morbidity pattern, average number of drugs prescribed, percentage of drugs prescribed by generic names and from the Essential drug list of Nepal (Essential drugs are those which satisfy the priority healthcare needs of the population), percentage of encounters with an antibiotic and an injection prescribed were noted.

Results: 1261 patients made 1772 visits. Upper respiratory tract infection and acid peptic disease were the most common diagnoses. The mean number of drugs was 1.99 . Only $19.5 \%$ and $39.6 \%$ of drugs were prescribed by generic name and from the Essential drug list. Antibiotics and injections were prescribed in $26.4 \%$ and $\mathbf{0 . 9 6 \%}$ of encounters. Cetrizine, vitamins, amoxicillin, the combination of paracetamol and ibuprofen and ranitidine were most commonly prescribed.

Conclusions: Upper respiratory tract infections and acid peptic disease were the common illnesses. Generic prescribing and use of essential drugs were low. Some of the drug combinations being used were irrational. Prescriber education may be helpful in encouraging rational prescribing.
\end{abstract}

KEYWORDS: Drug use review, drug utilization, morbidity patterns, outpatients

\section{INTRODUCTION}

Drug utilization research has been defined by the

*To whom correspondence should be addressed: Dr.P.Ravi Shankar Department of Pharmacology, Manipal College of Medical Sciences P.O.Box 155 Deep Heights Pokhara, Nepal.

Phone: 00977-61-523600.

Fax: 00977-61-522160.

E-mail:pathiyilravi@gmail.com,pathiyilravi@hotmail.com
World Health Organization (WHO) as "the marketing, distribution, prescription and use of drugs in a society, with special emphasis on the resulting medical, social and economic consequences" (1). The assessment of drug utilization is important for clinical, educational and economic purposes (2). Prescribing patterns need to be evaluated periodically to increase the therapeutic efficacy, decrease adverse effects and provide feedback 
to prescribers $(3,4)$. Drug utilization reviews are useful for obtaining information about drug use patterns and for identifying high cost drugs (5).

Irrational and inappropriate use of drugs is a major concern in both developed and developing countries $(6,7)$. The cost of irrational use of medicines is enormous in terms of scarce resources and the adverse clinical consequences of therapies that may have real risks but no objective benefits .

Previous studies in our hospital had shown deficiencies in use of medicines $(8,9)$. The problems observed were that most of the drugs were prescribed by brand names and the number of drugs prescribed from the Essential drug list was low. The World Health Organization (WHO) defines essential drugs as those that satisfy the health care needs of the majority of the population. They should therefore be available at all times in adequate amounts and in the appropriate dosage forms. Lately, the term 'drugs' has been replaced by 'medicines'. A number of interventions have been recently introduced at the Manipal Teaching hospital (MTH) to ensure more rational use of medicines (10). The terms 'inappropriate' and 'irrational' are often used interchangeably. The drugs chosen may not be appropriate to the patient, the diagnosis, economic status etc. The conference of experts on the rational use of drugs, convened by the WHO in Nairobi in 1985 defined that "Rational use of drugs requires that patient receive medication appropriate to their clinical needs, in doses that meet their own individual requirement for an adequate period of time and at the lowest cost to them and their community."

A previous study was carried out to obtain information on the prescribing patterns among medical outpatients (9). However, information on the morbidity pattern and prescribing patterns of drugs among out patients in other specialties are lacking in Western Nepal. A previous study was carried out around five years back with a rather limited time window, hence the usefulness of the present study. The objectives of the study were to evaluate prescribing patterns using drug use indicators and obtain information on the demographic characteristics and morbidity patterns of patients attending the outpatient department (OPD) during the study period. Information on the demographic characteristics and the morbidity patterns will help in determining what diseases were common in different age groups and genders. Proper prescribing habits and rational use of medicines can play a significant role in ensuring quality care in MTH and other healthcare facilities.

\section{METHODS}

The study was carried out at the Manipal Teaching hospital, a tertiary care hospital attached to the Manipal College of Medical Sciences, Pokhara, Nepal. The retrospective hospital record based study was carried out over a one year period of January $1^{\text {st }} 2004$ to December $31^{\text {st }} 2004$.

New patients attending the outpatient department (OPD) of MTH during the study period were considered for analysis. Follow up visits during the study period were included and were counted as separate visits. Visits for a new diagnosis by the selected patients during the study period were not considered. A total number of 32,017 patients attended the OPDs during the study period. Of this, 5\% (1600 cases) (1 in every 20 ) were selected by systematic random sampling. In systematic random sampling, first a number within the sampling interval (twenty in our study) is chosen using random number tables. We chose a random number between 1 and 20. Then every 20th patient following the first number chosen was selected from the outpatient register maintained in the medical records department (MRD).

Patient visiting the emergency department or who got admitted during the OPD visit were not included in the study. We expected that their prescribing patterns may be substantially different from those of ambulatory patients. Records not available in the medical records department were excluded from the study. After all these exclusions, a total of 1261 cases were analyzed.

The age, sex, address and occupation of the patients were noted. The diagnosis/diagnoses were noted and the prescribing patterns of the drugs were analyzed. The patients were grouped into the age groups of $<1$ year, 1 5 years, 5-15 years, 15-25 years, 25-35 years, 35-45 years, $45-55$ years, $55-65$ years and above 65 years. We selected this particular age distribution as we wanted to study the infants and under-five populations as a separate age group. Under-five morbidity and mortality is a major health problem in Nepal. The percentage of individuals in each age group was calculated. The commonly occurring classes of diseases according to the International Classification of Diseases (ICD 10) were noted. Most common diseases were found out for each age group.

The average number of drugs per encounter was calculated. The percentage of drugs prescribed from the Essential drug list of Nepal was calculated. The percentage of drugs prescribed by generic name was noted (11). The percentage of fixed-dose combinations (FDCs) prescribed was noted. The components of the FDCs were detailed. The ten most commonly prescribed group of drugs and individual drugs were detailed. The ten most commonly prescribed antibiotics were recorded. The percentage of encounters with an antibiotic and an injection prescribed were calculated. 
Among the antibiotics, the frequency of prescribing of different groups of antibiotics was studied. Among the FDCs the preparations containing at least one antibiotic, one non-steroidal anti-inflammatory drug (NSAID) or one corticosteroid or combinations of these were noted. These are common components of FDCs and many preparations contain these drugs either individually or in combination.

The relevant authorities were kept informed. However, ethical clearance was not obtained for the study. The data analysis was carried out manually. The data was expressed as percentage, mean and total numbers.

\section{RESULTS}

A total of 1261 files were analyzed. The catchment area of our hospital is Pokhara city, Kaski district in which Pokhara is situated and the neighboring districts of Syangja, Tanahu, Parbat and Baglung. The hospital also gets patients from the mountain districts of Mustang and Manang and the hill district of Gorkha.

Six hundred and eighty patients $(53.9 \%)$ were female. The age group 15-25 years accounted for the highest number [364 (28.9\%)] of patients. The age distribution of outpatients is shown in Figure 1. Only 909 patient files $(72.1 \%)$ contained information on the occupation of the patients. Students were the largest group to visit the OPD [398patients (43.8\% of the 909 patients)]. The distribution of patients by occupation has been shown in Table 1.

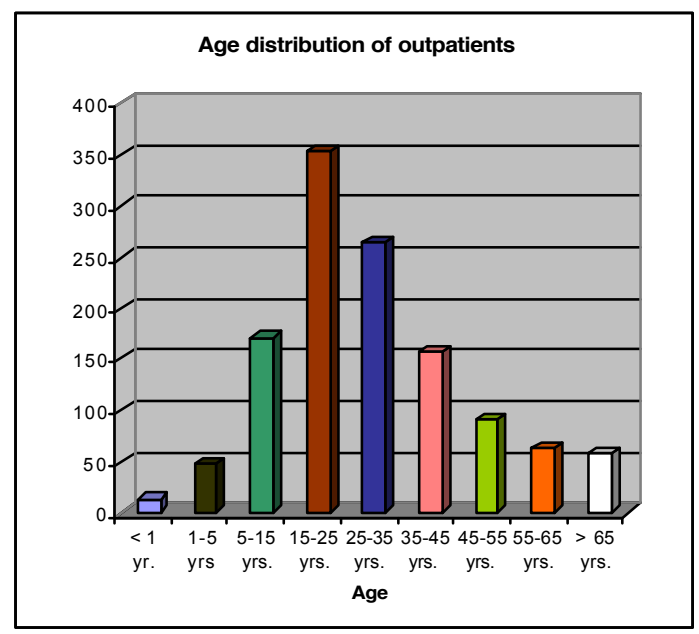

Figure 1. Age distribution of outpatients

The 1261 patients had made 1772 visits during the study period. Diseases of the skin and soft tissues [237 patients (18.8\%)] (which included tinea infection, acne vulgaris, scabies, and musculoskeletal pain) were the most common. Other groups of diseases in decreasing frequency were diseases of the digestive system (175 patients), diseases of the respiratory system (160 patients), and infectious and parasitic diseases (145 patients). Diseases of the genitourinary system (127 patients) were also common. Mental disorders and behavioral problems were seen in 31 patients. Many patients had more than one diagnosis. In cases of multiple diagnoses, the diagnosis/es for which treatment was given during the visit were noted. $15.96 \%$ of the files did not have any diagnosis written. The missing diagnoses were classified as 'diagnosis not written'. The common individual illnesses among outpatients are shown in Table 2. Going by the age group, tinea infection was the most common illness in the age group $<1$ year, impetigo in the 1-5 year age group, upper respiratory tract infection (URTI) was the most common illness in the 5-15, 15-25, 25-35 and 35-45 year age groups. Acid peptic disease (APD) was most common in the age group of 45-55 years and COPD in the age groups 55-65 years and more than 65 years.

The total number of drugs prescribed was 3532 . The oral dosage form accounted for $71.9 \%$ of drugs while $27.5 \%$ were prescribed in the topical form. The mean \pm SD number of drugs per prescription was $1.99 \pm 1.25$. Only $19.5 \%$ drugs were prescribed by generic names. At least one antibiotic was prescribed in 468 of the 1772 encounters $(26.4 \%)$. Seventeen encounters $(0.96 \%)$ had at least one injection prescribed. A total of 21 injections were prescribed. The common injections prescribed were tetanus toxoid followed by triamcinolone. Only 1399 drugs (39.6\%) were prescribed from the Essential drug list of Nepal.

Table 1. Distribution of patients by occupation

\begin{tabular}{lc}
\hline Occupation & $\begin{array}{c}\text { Number of patients } \\
\text { (percentage of total patients) } \mathbf{N = 9 0 9 *}\end{array}$ \\
\hline Students & $398(43.8)$ \\
\hline House wives & $208(22.9)$ \\
\hline Farmers & $148(16.3)$ \\
\hline Office job holders & $61(6.7)$ \\
\hline Others & $94(10.3)$ \\
\hline
\end{tabular}

* Only 909 patient files contained information on occupation

Groups of drugs which were commonly prescribed were antihistamines [251 prescriptions $(7.1 \%$ of the 3532 drugs prescribed)], NSAIDs (6.8\%) and anti ulcer drugs $(6.1 \%)$. The most commonly prescribed groups of drugs have been shown in Table 3 . The most frequently prescribed individual drugs were cetrizine [226 prescriptions $(6.4 \%)]$, multivitamin preparations (3.7\%), amoxicillin (3.1\%) and the FDC of paracetamol 
\& ibuprofen $(2.7 \%)$. The ten most commonly prescribed individual drugs are shown in Table 4.

Table 2. Most common diagnoses among outpatients

\begin{tabular}{lc}
\hline \multicolumn{1}{c}{ Diagnosis } & $\begin{array}{c}\text { \# of cases } \\
\text { (\% total patients) } \mathbf{N = 1 2 6 1 *}\end{array}$ \\
\hline Upper respiratory tract infections & $153(12.1)$ \\
\hline Acid peptic disease & $81(6.4)$ \\
\hline Tinea infection & $57(4.5)$ \\
\hline Acne vulgaris & $41(3.2)$ \\
\hline Scabies & $36(2.8)$ \\
\hline Musculoskeletal pain & $28(2.2)$ \\
\hline Conjunctivitis & $28(2.2)$ \\
\hline Otitis media & $28(2.2)$ \\
\hline Chronic obstructive pulmonary disease & $26(2.1)$ \\
\hline Dental caries & $26(2.1)$ \\
\hline
\end{tabular}

* Diagnosis was not available in the case record of 201 patients

Table 3. Most commonly prescribed groups of drugs among hospital outpatients

\begin{tabular}{lcc}
\hline Group of drugs & Number $(\%$ of total $)$ & $\mathbf{N}=\mathbf{3 5 3 2}$ \\
\hline Antihistaminics & 251 & $(7.1)$ \\
\hline NSAIDs & 248 & $(6.8)$ \\
\hline Anti ulcer drugs & 215 & $(6.1)$ \\
\hline Antifungal agents & 180 & $(5.1)$ \\
\hline Corticosteroids & 156 & $(4.4)$ \\
\hline Antidepressants & 82 & $(2.3)$ \\
\hline Drugs acting on skin + mucous membrane & 66 & $(1.9)$ \\
\hline Nasal decongestants & 65 & $(1.8)$ \\
\hline Antiprotozoals & 62 & $(1.75)$ \\
\hline Antihelminthics & 59 & $(1.7)$ \\
\hline
\end{tabular}

Antibiotics were a commonly prescribed group of drugs and accounted for 582 of the 3532 drugs (16.5\%) prescribed. The most commonly prescribed groups of antibiotics were the penicillin group [213 of the total of 582 antibiotics (36.6\%)] followed by quinolones $(19.6 \%)$, macrolides $(11.7 \%)$, tetracyclines $(8.8 \%)$, and aminoglycosides $(4.5 \%)$. The most commonly prescribed individual antibiotics were amoxicillin [111 prescriptions (3.1\% of the 3532 drugs prescribed)] followed by the fixed dose combination (FDC) of ampicillin and cloxacillin (2.3\%) and ciprofloxacin $(2.2 \%)$. Other commonly prescribed antibiotics were doxycycline, erythromycin and norfloxacin. The most commonly prescribed antibiotics are detailed in Table 5. FDCs accounted for 559 of the 3532 drugs (15.8\%) prescribed. Only $4.5 \%$ of FDCs were from the Essential drug list of Nepal. The most common FDCs were multivitamin preparations (3.7\% of the 3532 drugs), paracetamol and ibuprofen (2.7\%), cyproheptadine and tricholine citrate $(1.5 \%)$, clotrimazole and tinidazole (1\%) and a combination of chlorbutanol, polyvinyl alcohol and povidone iodine $(0.85 \%)$. These combinations were prescribed by brand names.

Table 4. Most commonly used drugs among hospital outpatients

\begin{tabular}{lcl}
\hline Drug & Number (\% of total) $\mathbf{N}=\mathbf{3 5 3 2}$ \\
\hline Cetrizine & 226 & $(6.4)$ \\
\hline Multivitamin preparations & 131 & $(3.7)$ \\
\hline Amoxicllin & 111 & $(3.1)$ \\
\hline Paracetamol + Ibuprofen & 95 & $(2.7)$ \\
\hline Ranitidine & 88 & $(2.5)$ \\
\hline Ampicillin + Cloxacillin & 83 & $(2.3)$ \\
\hline Diclofenac & 79 & $(2.2)$ \\
\hline Ciprofloxacin & 78 & $(2.2)$ \\
\hline Clotrimazole & 61 & $(1.7)$ \\
\hline Omeprazole & 57 & $(1.6)$ \\
\hline
\end{tabular}

Of the combination preparations, 110 of the 559 preparations contained at least one NSAID, 36 preparations contained at least one antibiotic while 31 preparations contained at least one corticosteroid.

The prescribing indicators among outpatients are shown in Table 6.

\section{DISCUSSION}

The age distribution of the patients showed that the age group of $15-25$ years constituted $28.9 \%$ of patients followed by the age group of 25-35 years and 5-15 years (Fig. 1). The distribution corresponds to that observed in the 2001 population census of Nepal (12). Young patients accounted for the majority of patients attending the OPD. The large population of young patients attending the OPD was also observed in a previous 
study (9). A possible reason could be the high proportion of young people in the population and also the fact that patients attending the emergency and who were admitted were not included. A greater proportion of older patients have been seen among in-patients in previous studies.

Table 5. Most commonly prescribed antibiotics among hospital outpatients

\begin{tabular}{lcl}
\hline Drug & \multicolumn{1}{l}{ Number (\% of total) } & $\mathbf{N}=3532$ \\
\hline Amoxicillin & 111 & $(3.1)$ \\
\hline Ampicillin + Cloxacillin & 83 & $(2.3)$ \\
\hline Ciprofloxacin & 78 & $(2.2)$ \\
\hline Doxycycline & 48 & $(1.4)$ \\
\hline Erythromycin & 35 & $(1)$ \\
\hline Norfloxacin & 21 & $(0.6)$ \\
\hline Fusidic acid & 19 & $(0.54)$ \\
\hline Clarithromycin & 16 & $(0.4)$ \\
\hline Sisomycin & 13 & $(0.36)$ \\
\hline Azithromycin & 11 & $(0.3)$ \\
\hline
\end{tabular}

Table 6. Prescribing indicators among outpatients

\begin{tabular}{|c|c|}
\hline Parameter & Value \\
\hline Total number of patients analyzed & 1261 \\
\hline $\begin{array}{l}\text { Total number of visits made for new diagnoses } \\
\text { during the period }\end{array}$ & 1772 \\
\hline Total number of drugs prescribed & 3532 \\
\hline Average number of drug per encounter & 1.99 \\
\hline$\%$ of drugs prescribed by generic name & 19.2 \\
\hline$\%$ of encounters with an antibiotic prescribed & 26.4 \\
\hline$\%$ of encounters with an injection prescribed & 0.96 \\
\hline $\begin{array}{l}\% \text { of drugs prescribed from the Essential drug } \\
\text { list of Nepal }\end{array}$ & 39.6 \\
\hline$\%$ of FDCs prescribed & 15.8 \\
\hline
\end{tabular}

Skin disease was the most common indication for visiting the OPD followed by diseases of the digestive and respiratory system. Infectious and parasitic diseases were common. The common individual diseases were URTI, APD, tinea infection, acne vulgaris and scabies. The common illnesses accounting for OPD visits in the
Western development region, in which Pokhara city is situated, were skin diseases, acute respiratory infections (ARI), diarrheal diseases, intestinal worms, gastritis and pyrexia of unknown origin (PUO) (13). In a study from a primary health centre in Duwakot near Kathmandu, viral fever, cut/injuries, hypertension, worm infestation and APD were the more common diseases (14).

The diseases listed above, with the exception of hypertension, are diseases of poverty and may be indicative of low socioeconomic development. Infectious diseases keep people in poverty. Worm infestation and respiratory diseases are common in poor developing countries. The big three infectious diseasesHIV/AIDS, TB and malaria claimed 5.7 million lives worldwide in 2001 (15). Ongoing ill health is a major reason why the poor are not able to break out of the cycle of poverty. Infections leads to poverty, poverty leads to infections (15).

The average number of drugs per prescription was 1.99. Our number is less than the 2.2 drugs per prescription noted in the Terai districts and the 2.1 drugs noted in the hill districts of Nepal (16). At the Manipal Teaching hospital, patients have to pay for their consultation, diagnostic tests or procedures and medicines. Poor in-patients often have their ward and laboratory charges waived and are supported by the Poor Patients' fund. These support facilities are not generally available to outpatients. In a previous study among medical outpatients, the mean number of drugs was 2.15 (9). In a study in a general hospital in Nigeria, the average number of drugs per prescription was 3.16 among outpatients (17). In Uzbekistan, rural primary physicians had prescribed 2.9 drugs per patient (18). The lower number of drugs noted in our hospital is a welcome sign and has to be encouraged. There may be an increased compliance, lower cost of therapy and decreased risk of drug interactions when lesser number of drugs are prescribed. However, we did not investigate whether diseases are being treated by alternative means and, we also did not look into the rationality of prescriptions. Thus, we could not conclude that the low number of drugs was not simply secondary to misuse or under treatment.

Only $19.2 \%$ of drugs in our study were prescribed by generic name. In a previous study, $32.6 \%$ of drugs were prescribed generically (9). Sarkar et al. had previously observed that $24.4 \%$ of drugs were prescribed by generic name (8). In previous studies, in other locations the percentage prescribed by generic name ranged from $38 \%$ to $51 \%(18,19)$. The decreasing percentage of drugs prescribed by generic names in our hospital is a matter of concern and the reasons for these should be investigated. Generic prescribing decreases the risk of wrong medicines being given to patients as many 
medicines with different generic names have similar brand names. Generic medicines are however, not being widely manufactured in Nepal. There is substantial price variation between brands and on prescribing by generic name, the pharmacist can dispense a cheaper brand reducing the cost of treatment.

Antibiotics and injections were prescribed in $26.4 \%$ and $0.96 \%$ of encounters respectively. Studies have shown that antibiotics and injections were prescribed in respectively $45.2 \%$ and $3.2 \%$ of encounters in the hill districts of Nepal (16). In a previous audit of prescriptions, antibiotics were prescribed in $47.8 \%$ of encounters (9). In Nigeria, antibiotics were prescribed in $50.3 \%$ of encounters (17). The lower number of encounters with an antibiotic or injection prescribed is a welcome sign and has to be encouraged (to be discussed subsequently). Also, only disposable needles are used in MTH and the risk of spread of blood borne infections has been reportedly decreased.

Only $39.6 \%$ of drugs were prescribed from the Essential drug list of Nepal. In a previous study, 62.5\% of drugs were prescribed from the Essential drug list (11). In a previous study at primary healthcare facilities in the Kaski district, the percentage of drugs prescribed from the Essential drug list varied from $70.9 \%$ to $74 \%$ (20). The low rate of prescribing of essential drugs is a matter of concern. Excessive use of multivitamin and combination preparations may be one of the factors responsible. The use of the antihistamine cetrizine, which is not on the Essential drug list, may be another contributory factor. It must be noted though that Essential drugs are primarily meant for primary healthcare systems while we studied drug utilization in a tertiary care hospital.

The most commonly used antibiotics were amoxicillin, FDC of ampicillin and cloxacillin and ciprofloxacin. In a previous study, the most frequently prescribed antibiotics were ampicillin, chloramphenicol and gentamicin (21). In medical outpatients, the most commonly prescribed antibiotics were amoxicillin, metronidazole, the FDC of metronidazole and diloxanide furoate and norfloxacin (9).

The antibiotics used in our hospital were older generation antibiotics and this is to be welcomed. However, the use of the FDC of ampicillin and cloxacillin is a matter of concern. The use of antibiotics should be in accordance with the sensitivity patterns of microorganisms in the particular area (22). If the organisms are sensitive to older antibiotics they should be used. The newer antibiotics are expensive and patients may not be able to afford a full course and they may opt for a truncated course increasing the likelihood of resistance. The newer antibiotics should be kept in reserve. Also, more data is available for older antibiotics which have been used in a larger number of patients and for a longer period of time. The FDC of ampicllin and cloxacillin often does not contain the requisite amount of each individual antibiotic. The combination is not synergistic as cloxacillin is not active against gram negative bacteria and does not inhibit beta lactamase while ampicillin is not active against staphylococci. Thus, the combination only adds to the cost and adverse effects of both drugs.

Antibiotics, antihistamines, NSAIDs, anti-ulcer drugs and corticosteroids were the group of drugs most commonly prescribed. In a previous study, antimicrobials, analgesics, and cough and cold remedies were most commonly prescribed (9). In a study in Duwakot health center, antipyretics, antibiotics and NSAIDs were most commonly prescribed (14). The use of drugs was in accordance with the high prevalence of infectious, skin and parasitic diseases among outpatients in our study.

FDCs accounted for $15.8 \%$ of drugs prescribed. In a previous study, FDCs accounted for $47 \%$ of drugs (9) while in Uttaranchal, India, FDCs accounted for 59\% of drugs prescribed (19). However, a problem noted was the use of irrational drug combinations in a few instances. Irrational combinations are those which contain a combination of drugs which may be used to treat a condition without arriving at a proper diagnosis. Combinations of antifungals, antibiotics and corticosteroids are available especially among dermatological preparations. The FDC may not contain the requisite amount of each individual drug and the combination may not be synergistic. Moreover, the FDCs were usually prescribed by brand name and this may be another factor responsible for the low percentage of drugs prescribed by generic names.

In Pokhara city, most medicines are available. Also the hospital pharmacy maintains adequate inventory and ensures adequate supply of medicines during the frequent bandhs (a form of political protest) and blockades. The hospital drug and therapeutics committee (DTC) has already taken note of the results of this and other studies. Generic prescribing is being strongly encouraged, the number of brands available in the pharmacy has been limited and newer drugs can be introduced in the hospital only after approval by the DTC. The information obtained from this study gives base line data on prescribing to outpatients and will be helpful to evaluate the effect of future and ongoing interventions, both managerial and educational.

Our study had many limitations. The mean cost of drugs and the mean duration of prescription were not calculated. The diagnosis was not available in a substantial number of prescriptions. The rationality of prescriptions was not looked into. The study was 
retrospective and non-drug treatments for different conditions were not analyzed. The study population was young. The results may not be comparable to those obtained from other centers where the patients may be sicker and on multiple medicines. A prospective study interviewing the outpatients may be considered. These limitations may impact on the generalizability of the results obtained.

\section{CONCLUSION}

Infectious diseases and parasitic infestations were common and this may be associated with low socioeconomic development. The average number of drugs per prescription was low but prescribing by generic names has to be encouraged. The use of essential drugs was low and the FDCs used were not rational in some cases.

Framing of standard treatment guidelines for common diseases and prescriber education regarding rational use of medicines as has been done in many hospitals may be helpful.

\section{ACKNOWLEDGMENT}

The help of Mr. Binu VS, Biostatistician at the Manipal College of Medical Sciences in the planning aspects of the study is gratefully acknowledged.

\section{REFERENCES}

1. World Health Organization. Introduction to drug utilization research. Oslo: 2003.

2. Uppal R, Nayak P, Sharma PL. Prescribing trends in internal medicine. Int J Clin Pharm Ther Toxicol 1984; 22:373-376.

3. Krishnaswamy K, Dinesh Kumar B, Radhaiah G. A drug use survey- precepts and practice. Eur J Clin Pharmacol 1985; 29:363-370.

4. Pradhan SC, Shewade DG, Shashindran CH, Bapna JS. Drug utilization studies. National Med J India 1988; 1:185-189.

5. Marshner JP, Thurmann P, Harder S, Rietbrock N. Drug utilization review on a surgical intensive care unit. Int J Clin Pharmacol Ther 1994; 32:447-451.
6. Hogerzeil HV. Promoting rational prescribing: an international perspective. Br J Clin Pharmacol 1995; 39:1-6.

7. Joshi MP. Rational prescribing. In: Joshi MP, Adhikari RK, eds. Manual of drugs and therapeutics. Kathmandu: Health Learning Materials Centre 1996:1-10.

8. Sarkar C, Das B. Prescribing trends in a teaching hospital in Western Nepal. Journal of Nepalgunj Medical College 2:4-5; 2002.

9. Shankar PR, Partha P, Nagesh S. Prescribing patterns in medical outpatients. Int J Clin Pract 2002; 56:549-551.

10. Mishra P. Enhancement of consumer safety and rational use of drug: an important role of Drug and Therapeutics Committee (DTC). HAI News 2005; 132:21-22.

11. Department of Drug Administration. National list of essential drugs, Nepal (Third revision). Kathmandu: 2002.

12. Central Bureau of Statistics. Population monograph of Nepal. Volumes I and II. Kathmandu:2003.

13. Department of Health Services. Annual Report 2058/59 BS (2001/2002 AD). Kathmandu: 2003.

14. Bajracharya S, Pandey S, Shakya YL. Drug prescribing pattern and disease pattern in KMC Duwakot health center. Kathmandu University Medical Journal 2004; 2:35-42.

15. World Health Organization. Scaling up the response to infectious diseases - A way out of poverty. Report on infectious diseases 2002. WHO/CDS/2002.7. Geneva: 2002.

16. INRUD, Nepal. 18th National training course on rational use of drugs. 15-20 April 2005. Kathmandu: 2005.

17. Chukwuani CM, Onifade M, Sumonu K. Survey of drug use practices and antibiotic prescribing pattern at a general hospital in Nigeria. Pharm World Sci 2002; 24:188-195.

18. Pavin M, Nurgozhin T, Hafner G, Yusufy F, Laing R. Prescribing practices of rural primary health care physicians in Uzbekistan. Trop Med Int Health 2003; 8:182-190.

19. Rishi RK, Sangeeta A, Surendra K, Tailang M. Prescription audit: experiences in Garhwal (Uttaranchal), India. Trop Doct 2003; 33:76-79.

20. Shankar R, Kumar P, Rana M, Dubey A, Shenoy N. A comparative study of drug utilization at different levels of the primary health care system in Kaski district, Western Nepal. N Z Med J 2003: 116.

21. Orrett FA. Antimicrobial prescribing patterns at a rural hospital in Trinidad: evidence for intervention measures. Afr J Med Sci 2001; 30:161-164.

22. World Health Organization. WHO global strategy for containment of antimicrobial resistance. WHO/CDS/CSR/DRS/2001.2. Geneva: 2001.

Dronacharya Lamichhane is a seventh semester medical student studying at the Manipal College of Medical Sciences, Nepal. He has a special interest in psychiatry.

Om Krishna Pathak is a seventh semester medical student studying at Manipal College of Medical Sciences, Nepal. He has special interests in public health research, particularly maternal and child health.

Bishnu Rath Giri is a seventh semester student at Manipal College of Medical Sciences, Nepal. He is interested in issues of community health and the measures to alleviate the health status, especially in rural areas. He is author of several publications related to health and the community.

Om Biju Panta is a seventh semester student at Manipal College of Medical Sciences, Nepal. He is interested in academics, pure and applied science researches and public health activities such as health education and awareness programs. He wants to pursue his future education in genetics and gene therapy.

Dr.P.Ravi Shankar is Assistant Professor of Pharmacology at the Manipal college of Medical Sciences, Pokhara, Nepal. His research interests are the teaching and learning of communication skills, as well as the teaching of rational use of medicines and pharmacoepidemiology. He is author of numerous publications in national and international journals. 